\title{
Associação dos fatores demográficos, clínicos e do manejo terapêutico no desfecho de pacientes sépticos atendidos em uma emergência hospitalar
}

Association of demographic, clinical and therapeutic management factors with the outcome of septic patients treated in a hospital emergency

Associacóin de factores demográficos, clínicos y do manejo terapéutico en el resultado de pacientes sépticos tratados en una emergencia hospitalar

\section{Rayssa Thompson Duarte ${ }^{\mathrm{I}}$, Ana Paula Amestoy de Oliveira' ${ }^{\mathrm{II}}$, Miriane Melo Silveira Moretti ${ }^{\mathrm{II}}$ Janete de Souza Urbanetto ${ }^{\text {IV }}$}

Resumo: Objetivo: identificar a associação dos aspectos demográficos, clínicos e do manejo terapêutico no desfecho dos pacientes diagnosticados com sepse em uma emergência hospitalar. Método: documental retrospectivo, 312 prontuários de pacientes sépticos, realizado análise descritiva, comparações de dados categóricos utilizaram-se os Testes de Qui quadrado ou Exato de Fisher, com correção de Monte Carlo. Resultados: idade média $65( \pm 17,66)$ anos, $51,3 \%$ eram mulheres, 52,6\% hipertensos, $28,5 \%$ diabéticos, $27,2 \%$ cardiopatas, $74 \%$ diagnosticados com sepse 52,3\% foco infeccioso pulmonar. Existe associação entre as variáveis demográficas e os desfechos $(\mathrm{p}<0,05)$. Histórico de acidente vascular encefálico, cirrose, cardiopatia e choque séptico associam-se ao óbito $(\mathrm{p}<0,05)$. Sinais de alerta e disfunções orgânicas avaliadas associam-se ao desfecho. Foco infeccioso $(\mathrm{p}=1)$ e taquicardia $(\mathrm{p}=0,823)$ não tem associação com desfecho nem com gravidade da sepse $(\mathrm{p}=0,120)$. Conclusão: há associação entre o perfil demográfico do paciente, comorbidades, disfunções orgânicas e manejo terapêutico, com a gravidade da sepse e o desfecho.

Descritores: Sepse; Serviço hospitalar de emergência; Perfil de saúde; Enfermagem

Abstract: Aim: To identify the association of demographic, clinical and therapeutic management aspects with the outcome of patients diagnosed with sepsis in a hospital emergency. Method: retrospective documentary, 312 records of septic patients, descriptive analysis, comparisons of categorical data were used using Chi-square or Fisher's exact tests, with Monte Carlo correction. Results: mean age $65( \pm 17.66)$ years, $51.3 \%$ were women, 52.6\%

\footnotetext{
${ }^{\mathrm{I}}$ Enfermeira assistencial. Especialista em urgência e emergência pelo Programa de Residência Multiprofissional da Pontifícia Universidade Católica do Rio Grande do Sul. Universidade Federal de Ciências da Saúde de Porto Alegre, Porto Alegre, RS, Brasil. rayssatd@hotmail.com. ORCID: 0000-0001-9443-8449

II Enfermeira assistencial. Mestre em Ensino na Saúde pela Universidade Federal de Ciências da Saúde de Porto Alegre. Pontifícia Universidade Católica do Rio Grande do Sul, Porto Alegre, RS, Brasil. anapamestoy@gmail.com. ORCID: 0000-0003-0986-6831

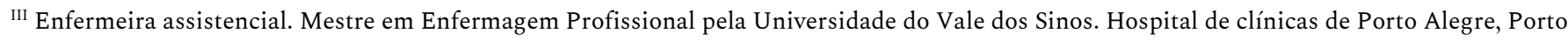
Alegre, RS, Brasil. miriane.moretti@hcpa.edu.br ORCID: 0000-0002-5365-5997

IV Professora do Curso de Enfermagem da Escola de Ciências da Saúde. Doutora em ciências da Saúde pela PUCRS. Pontifícia Universidade Católica do Rio Grande de Sul, Porto Alegre, RS, Brasil. jurbanetto@pucrs.br. ORCID: http://orcid.org/0000-0002-4697-1641
} 
Associação dos fatores demográficos, clínicos e do manejo terapêutico ao desfecho de pacie... $\mid 2$

hypertensive, $28.5 \%$ diabetic, $27.2 \%$ heart disease, $74 \%$ diagnosed with sepsis $52.3 \%$ infectious pulmonary focus. There is an association between demographic variables and outcomes $(\mathrm{p}<0.05)$. History of stroke, cirrhosis, heart disease, and septic shock are associated with death $(\mathrm{p}<0.05)$. Warning signs and organ dysfunction evaluated are associated with the outcome. Infectious focus $(\mathrm{p}=1)$ and tachycardia $(\mathrm{p}=0.823)$ has no association with outcome or severity of sepsis $(\mathrm{p}=0.120)$. Conclusion: there is an association between patient demographic profile, comorbidities, organ dysfunction and therapeutic management, with sepsis severity and outcome.

Descriptors: Sepsis;Emergency Service, Hospital; Health Profile; Nursing

Resumen: Objetivo: identificar la asociación de los aspectos demográficos, clínicos y terapeuticos en el resultado de pacientes diagnosticados con sepsis en una emergencia hospitalar. Método: documental retrospectivo, se utilizaron 312 registros de pacientes sépticos, análisis descriptivo, comparaciones de datos categóricos utilizando Chi-cuadrado o pruebas exactas de Fisher, con corrección de Monte Carlo. Resultados: edad media 65 ( \pm 17.66 ) años, $51.3 \%$ eran mujeres, $52.6 \%$ hipertensos, $28.5 \%$ diabéticos, $27.2 \%$ enfermedades del corazón, $74 \%$ diagnosticados con sepsis 52.3\% foco pulmonar infeccioso. Existe una asociación entre las variables demográficas y los resultados $(\mathrm{p}<0.05)$. Los antecedentes de accidente cerebrovascular, cirrosis, enfermedad cardíaca y shock séptico están asociados con la muerte $(\mathrm{p}<0.05)$. Las señales de advertencia y la disfunción orgánica evaluada están asociadas con el resultado. El foco infeccioso $(\mathrm{p}=1)$ y la taquicardia $(\mathrm{p}=0.823)$ no tienen asociación con el resultado o la gravedad de la sepsis $(\mathrm{p}=0.120)$. Conclusión: existe una asociación entre el perfil demográfico del paciente, las comorbilidades, la disfunción orgánica y el tratamiento terapéutico, con la severidad y el resultado de la sepsis.

Descriptores: Sepsis; Servicio de Urgencia em Hospital; Perfil de salud; Enfermería

\section{Introdução}

Diversas patologias e comorbidades são conhecidas por desencadearem disfunções orgânicas que levam a um declínio agudo do estado de saúde do paciente, fazendo-o procurar um atendimento de urgência e emergência. Casos de pacientes politraumatizados, cardiopatas com infarto agudo do miocárdio (IAM) ou acidentes vasculares encefálicos (AVE) são os principais responsáveis pelos atendimentos de emergência. Entretanto, tem-se vivenciado uma equiparação da incidência dessas doenças com os casos de pacientes com sepse, que afetam milhões de pessoas, matando um a cada quatro pacientes. ${ }^{1}$ Os casos de sepse têm adquirido notoriedade, mesmo com a subnotificação no diagnóstico ou quando a causa do óbito é atribuída erroneamente à doença de base e não à sepse. ${ }^{2}$

De acordo com o último Guidelines for the Management of Severe Sepsis and Septic Shock, publicado em 2017, o conceito de sepse é entendido por um órgão acometido por uma 
3 | Duarte RT, Oliveira APA, Moretti MMS, Urbanetto JS

ameaça vital, causada por uma resposta desordenada a infecção. ${ }^{1}$ Enquanto o choque séptico define-se como um agravo da sepse, comprometendo o sistema circulatório, celular e metabólico, com necessidade de infusão de drogas vasopressoras, com um risco maior de mortalidade. ${ }^{1}$

Recentemente novas definições de sepse foram publicadas, alterando a definição para “presença de disfunção orgânica ameaçadora à vida secundária à resposta desregulada do organismo à infecção” e o diagnóstico passou a ser associado a dois ou mais pontos no escore do Sequential Organ Failure Assessment (SOFA). ${ }^{3}$ Esse estudo manteve o uso dos critérios de definição anteriores, pois a Associação de Medicina Intensiva Brasileira (AMIB) e o Instituto Latino Americano de Sepse (ILAS) consideraram que o uso dos critérios de SOFA em países subdesenvolvidos, levando em conta seus perfis de saúde, poderia sensibilizar excessivamente o diagnóstico retardando o tratamento adequado aos casos diagnosticáveis. ${ }^{4}$

As medidas de reconhecimento das disfunções orgânicas e o tratamento dessa patologia também são reconhecidas e descritas por diretrizes como a da Surviving Sepsis Campaign, que sofreu uma atualização organizando suas ações nas primeiras horas do atendimento em bundles. As ações que compõem o manejo do paciente compreendem: coleta de lactato sérico, coleta de hemocultura antes do início da antibioticoterapia, início de antibióticos o mais precoce possível, reposição volêmica, uso de vasopressores (nos casos que não tiverem resposta à reposição volêmica e mantiverem pressão arterial média $(\mathrm{PAM})<65 \mathrm{mmHg})$, mensuração da pressão venosa central (PVC), mensuração da saturação venosa de oxigênio, reavaliação dos níveis de lactato em pacientes com hiperlactatemia inicial. ${ }^{2-3} \mathrm{~A}$ excessiva sensibilidade e a não especificidade relacionada aos critérios diagnósticos relacionados à sepse, as comorbidades do paciente e o não seguimento das diretrizes de tratamento, dificultam o atendimento precoce, influenciando negativamente no prognóstico do paciente ${ }^{4}$ 
Associação dos fatores demográficos, clínicos e do manejo terapêutico ao desfecho de pacie... $\mid 4$

Sabe-se que algumas condições particulares do paciente podem influenciar na resposta de seu organismo ao combate à sepse. Dentre estes fatores, destacam-se a relação do gênero e das comorbidades com o desfecho relacionado à mortalidade evidenciada por estudos epidemiológicos. Evidencia-se a importância da identificação do perfil desses pacientes para o desenvolvimento de protocolos e políticas públicas mais eficazes. ${ }^{5}$

Percebe-se a importância de estudos sobre sepse e choque séptico para a identificação precoce, proatividade e adesão às ações relacionadas ao manejo do paciente com sepse no serviço de emergência. Desta forma, a questão de pesquisa deste estudo foi: quais fatores estão associados ao agravamento da sepse e ao desfecho dos pacientes com sepse? O objetivo estabelecido foi identificar a associação dos aspectos demográficos, clínicos e do manejo terapêutico ao desfecho dos pacientes diagnosticados com sepse em uma emergência hospitalar.

\section{Método}

Pesquisa documental do tipo coorte retrospectiva, desenvolvida em um Serviço de Emergência de um hospital universitário de Porto Alegre. A população foi composta por 587 prontuários de pacientes adultos diagnosticados com sepse ou choque séptico, atendidos no hospital, durante 01 de janeiro a 31 de dezembro de 2014. A amostra foi composta por 312 pacientes diagnosticados com sepse ou choque séptico na emergência. Excluíram-se 275 pacientes diagnosticados em outro setor hospitalar.

Os dados foram extraídos de um banco de dados do Serviço de Controle de Infecção do hospital, construído a partir da busca ativa, notificação de inclusão no protocolo de sepse ou de prontuários do paciente. Este banco contempla variáveis demográficas (idade e sexo), variáveis relacionadas às comorbidades (diagnósticos médicos), aos sinais de alerta (hipertermina, hipotermina, taquipneia, taquicardia, hipotensão, alteração de sensório, rigidez de nuca, leucocitose/leucopenia e presença de bastonados), e as disfunções orgânicas relacionadas a sepse 
5 | Duarte RT, Oliveira APA, Moretti MMS, Urbanetto JS

(alteração de saturação de oxigênio (SpO2), PAM<65mmHg, queda de pressão arterial, Lactato $>2$, razão normalizada internacional $(\mathrm{RNI})<1,5$, plaquetopenia, bilirrubinas $>2$, alteração de creatinina, alteração de pressão parcial de gás carbônico (PCO2), e a adesão ao protocolo de reconhecimento da sepse e as intervenções terapêuticas realizadas (administração de antibioticoterapia, coleta de hemocultura, pacote de exames da sepse - gasometria arterial e venosa, hemograma, creatinina, plaquetas, lactato, bilirrubinas e tempo de protrombina (TP)-, reposição volêmica, instalação e PVC, punção de cateter venoso central (CVC), sondagem vesical de demora (SVD) e tubo orotraqueal (TOT).

Os desfechos do estudo foram alta/óbito/internação em até 14 dias (D14) e alta/óbito entre 15 e 30 dias (D15 a 30). A gravidade descrita foi relacionada a sepse e choque séptico. Para cada paciente incluído no estudo, foram coletados dados desde o primeiro dia da internação até a alta ou óbito do paciente.

Para análise estatística foi utilizado o programa Statistical Package for the Social Sciences (SPSS), versão 20.0 (SPSS Inc., Chicago, IL, EUA. 2010). As variáveis contínuas foram descritas pela média e desvio-padrão e as variáveis categóricas por frequência simples e percentual. Para as comparações de dados categóricos foram utilizados os Testes de Qui quadrado ou Exato de Fisher, com correção de Monte Carlo. Foi adotado o nível de significância de $5 \%$.

Este artigo está vinculado ao projeto de pesquisa intitulado “A Sepse Grave no Contexto Hospitalar e o Conhecimento de Profissionais e Acadêmicos da Área da Saúde e do Público Leigo”, aprovado pelo Comitê de Ética e Pesquisa da instituição em 9 de maio de 2015, sob Certificado de Apresentação para Apreciação Ética nº 44458215.9.0000.5336, respeitando todas as exigências éticas e científicas, baseadas nas disposições da Resolução $\mathrm{n}^{\circ} 466 / 2012$ do Ministério da Saúde, acerca das diretrizes e normas para pesquisas envolvendo seres humanos. 
Associação dos fatores demográficos, clínicos e do manejo terapêutico ao desfecho de pacie... I 6

\section{Resultados}

O perfil dos pacientes, compreendia indivíduos com média de idade de $65 \pm 17,66$ anos e maior distribuição do sexo feminino 160 (51,3\%). Quanto às comorbidades apresentadas, 164 (52,6\%) eram hipertensos, $89(28,5 \%)$ diabéticos, $85(27,2 \%)$ tinham doença cardiovascular, 56 (17,9\%) Doença Pulmonar Obstrutiva Crônica (DPOC), 52 (16,7\%) neoplasias, 47 (15,1\%) histórico de AVE, 42 (13,5\%) Doença Renal Crônica (DRC), 23 (7,4\%) eram imunossupressos, 14 (4,5\%) viviam com Vírus da imunodeficiência humana (HIV), 11 (3,5\%) eram transplantados prévios e 6 (1,9\%) apresentavam doença hepática. Quanto à gravidade da sepse, haviam 231 (74\%) pacientes com diagnóstico de sepse com disfunção orgânica e 81 (26\%) em choque séptico. Em relação ao foco infeccioso, 162 (51,9\%) eram de foco respiratório, 68 (21,8\%) de foco urinário, 41 (13,1\%) de foco abdominal, 24 (7,69\%) outras infecções, 11 (3,5\%) de partes moles e 6 (1,9\%) não possuíam foco definido.

As associações encontradas entre as variáveis relacionadas à faixa etária, sexo e as comorbidades dos pacientes com o desfecho dos pacientes com sepse e choque séptico, atendidos na unidade de emergência, estão descritas na Tabela 1.

Tabela 1: Associação entre faixa etária, sexo, comorbidades de pacientes com sepse ou choque séptico e o desfecho apresentado no ano de 2014 (n=312). Porto Alegre, RS, Brasil, 2015.

Desfecho em até 14 dias

Desfecho 15 a 30 dias

\begin{tabular}{|c|c|c|c|c|c|c|c|}
\hline \multirow[t]{2}{*}{ Variáveis } & Óbito & Internação & Alta & \multirow{2}{*}{$\mathrm{P}$} & Óbito & Alta & \multirow{2}{*}{$\mathrm{P}$} \\
\hline & n $(\%)$ & n $(\%)$ & n $(\%)$ & & n $(\%)$ & n $(\%)$ & \\
\hline \multicolumn{8}{|l|}{ Faixa etária } \\
\hline 18 a 56 anos & $21(20,8)$ & $2(2,0)$ & $78(77,2)$ & & $2(2,5)$ & $78(97,5)$ & \\
\hline 57 a 71 anos & $28(26,9)$ & $5(4,8)$ & $71(68,3)$ & 0,047 & $05(6,6)$ & $71(93,4)$ & 0,015 \\
\hline 72 a 95 anos & $25(23,4)$ & $12(11,2)$ & $70(65,4)$ & & $12(14,6)$ & $70(85,4)$ & \\
\hline
\end{tabular}


7 | Duarte RT, Oliveira APA, Moretti MMS, Urbanetto JS

\begin{tabular}{|c|c|c|c|c|c|c|c|}
\hline Sexo & & & & & & & \\
\hline Feminino & $33(20,6)$ & $06(3,8)$ & $121(75,6)$ & & $06(4,7)$ & $121(95,3)$ & \\
\hline Masculino & $41(27,0)$ & $13(8,6)$ & $98(64,5)$ & 0,059 & $03(11,7)$ & $98(88,3)$ & 0,047 \\
\hline Comorbidades & & & & & & & \\
\hline AVE prévio & $10(21,3)$ & $07(14,9)$ & $30(63,8)$ & 0,040 & $07(18,9)$ & $30(81,1)$ & 0,015 \\
\hline DPOC & $12(21,4)$ & $03(5,4)$ & $42(73,2)$ & 0,934 & $03(6,8)$ & $41(93,2)$ & 1,000 \\
\hline Neoplasia & $12(23,1)$ & $05(9,6)$ & $35(67,3)$ & 0,520 & $05(12,5)$ & $35(87,5)$ & 0,332 \\
\hline Transplante & $01(9,1)$ & - & $10(90,9)$ & 0,460 & - & $10(100,0)$ & 1,000 \\
\hline Imunodeprimido & $06(26,1)$ & $01(4,3)$ & $16(69,6)$ & 0,929 & $01(5,9)$ & $16(94,1)$ & 1,000 \\
\hline Diabete Mellitus & $19(21,3)$ & $02(2,2)$ & $68(76,4)$ & 0,135 & $02(2,9)$ & $68(97,1)$ & 0,060 \\
\hline Cirrose & $04(66,7)$ & - & $2(33,3)$ & 0,043 & - & $02(100)$ & 1,000 \\
\hline DRC & $12(28,6)$ & $02(4,8)$ & $28(66,7)$ & 0,739 & $02(6,7)$ & $28(93,3)$ & 1,000 \\
\hline HAS & $43(26,2)$ & $13(7,9)$ & $108(65,9)$ & 0,153 & $03(10,7)$ & $108(89,3)$ & 0,110 \\
\hline Cardiopatia & $28(32,9)$ & $7(8,2)$ & $50(58,8)$ & 0,027 & $7(12,3)$ & $50(87,7)$ & 0,171 \\
\hline HIV Positivo & $4(28,6)$ & $1(7,1)$ & $9(65,3)$ & 0,711 & $1(10,0)$ & $9(90,0)$ & 0,572 \\
\hline
\end{tabular}

As associações entre o foco infeccioso, sepse ou choque séptico, sinais de alerta e disfunções orgânicas apresentadas pelos pacientes sépticos e sua associação com os desfechos está apresentada na Tabela 2.

Tabela 2: Associação entre foco infeccioso, gravidade da sepse, sinais de alerta, disfunções orgânicas e o desfecho apresentados por pacientes sépticos em uma unidade de emergência no ano de 2014 ( $\mathrm{n}=312)$. Porto Alegre, RS, Brasil, 2015.

Desfecho em até 14 dias

\begin{tabular}{cccccc}
\hline Óbito & Internação & Alta & & Óbito & Alta \\
$n$ & $\mathrm{n}(\%)$ & $\mathrm{n}(\%)$ & & $\mathrm{n}(\%)$ & $\mathrm{n}(\%)$
\end{tabular}

\section{Foco}


Associação dos fatores demográficos, clínicos e do manejo terapêutico ao desfecho de pacie... $\mid 8$

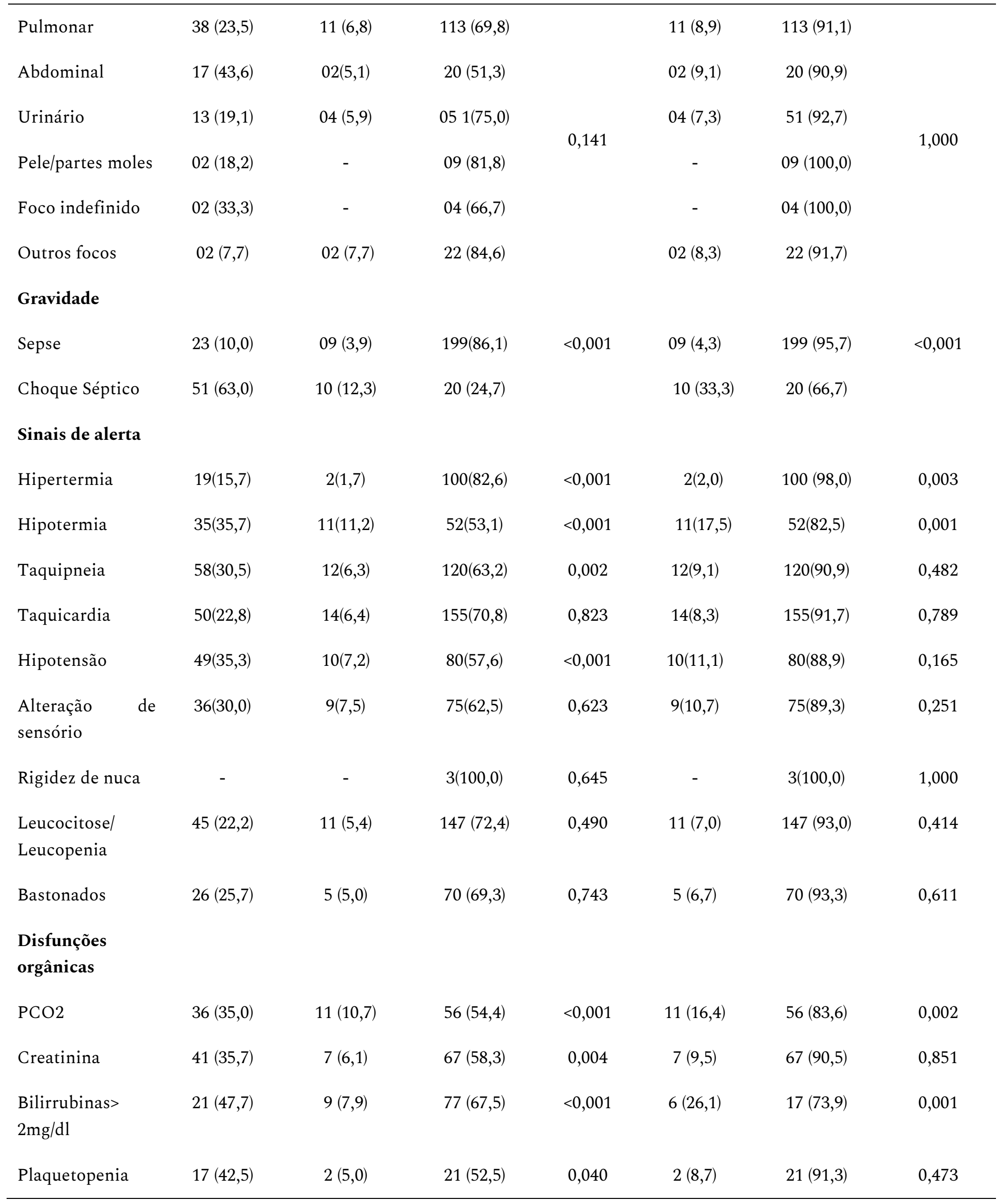


9 | Duarte RT, Oliveira APA, Moretti MMS, Urbanetto JS

\begin{tabular}{lcccccc}
\hline RNI $>1,5$ & $24(57,1)$ & $2(4,8)$ & $16(38,1)$ & $<0,001$ & $2(11,1)$ & $16(88,9)$ \\
$\begin{array}{l}\text { Lactato } \\
\text { mmol/L }\end{array}$ & $51(37,2)$ & $14(10,2)$ & $72(52,6)$ & $<0,001$ & $14(16,3)$ & $72(83,7)$ \\
Queda de PAS & $26(33,8)$ & $9(11,7)$ & $42(54,5)$ & 0,002 & $9(17,6)$ & $42(82,4)$ \\
PAM<65mmHg & $52(37,4)$ & $11(7,9)$ & $76(54,7)$ & $<0,001$ & $11(12,6)$ & $76(87,4)$ \\
SPO2 & $64(27,8)$ & $16(7,0)$ & $150(65,2)$ & 0,020 & $16(9,6)$ & 0,044 \\
\hline
\end{tabular}

Os dados apresentados na Tabela 3 demonstram as associações entre variáveis relacionadas à faixa etária, sexo, comorbidades e foco infeccioso com o diagnóstico de gravidade da sepse.

Tabela 3: Associação entre a gravidade da sepse e variáveis relacionadas à faixa etária, sexo, comorbidades e foco infeccioso do paciente em uma unidade de emergência no ano de 2014 $(\mathrm{n}=312)$. Porto Alegre, RS, Brasil, 2015.

\begin{tabular}{lcc}
\hline \multirow{2}{*}{ Variável } & Sepse & Choque Séptico \\
\cline { 2 - 3 } & $\mathrm{n}(\%)$ & $\mathrm{n}(\%)$ \\
\hline
\end{tabular}

Idade

\begin{tabular}{|c|c|c|}
\hline 18 a 56 anos & $82(81,2)$ & $19(18,8)$ \\
\hline 57 a 71 anos & $69(66,3)$ & $35(33,7)$ \\
\hline 72 a 95 anos & $80(74,8)$ & $27(25,2)$ \\
\hline
\end{tabular}

Sexo

Feminino

Masculino

Comorbidades

AVE prévio

$31(66,0)$

$40(71,4)$

$37(71,2)$

DPOC

Neoplasia
$26(16,3)$

$<0,001$

$97(63,8)$

$55(36,2)$

$16(34,0)$

0,170

$16(28,6)$

0,623

$15(28,8)$

0,603 
Associação dos fatores demográficos, clínicos e do manejo terapêutico ao desfecho de pacie... I 10

\begin{tabular}{|c|c|c|c|}
\hline Transplante & $9(81,8)$ & $2(18,2)$ & 0,734 \\
\hline Imunodeprimido & $16(69,6)$ & $7(30,4)$ & 0,611 \\
\hline Diabetes Mellitus & $68(76,4)$ & $21(23,6)$ & 0,547 \\
\hline Cirrose & $2(33,3)$ & $4(66,7)$ & 0,041 \\
\hline DRC & $32(76,2)$ & $19(23,8)$ & 0,732 \\
\hline Hipertensão Arterial Sistêmica & $118(72,0)$ & $46(28,0)$ & 0,376 \\
\hline Cardiopata & $58(68,2)$ & $27(31,8)$ & 0,153 \\
\hline & & & 0,372 \\
\hline HIV Positivo & $12(85,7)$ & $2(14,3)$ & \\
\hline \multicolumn{4}{|l|}{ Foco } \\
\hline Pulmonar & $121(74,7)$ & $41(25,3)$ & \\
\hline Abdominal & $22(56,4)$ & $17(43,6)$ & \\
\hline Urinário & $52(76,5)$ & $16(23,5)$ & \\
\hline & & & 0,120 \\
\hline Pele/partes moles & $9(81,8)$ & $2(18,2)$ & \\
\hline Foco indefinido & $6(100,0)$ & - & \\
\hline Outros focos & $21(80,8)$ & $5(19,2)$ & \\
\hline
\end{tabular}

Não apresentaram associação com o desfecho do paciente com sepse em 14 dias ou entre 15 e 30 dias o número de comorbidades (p=0,207), a adesão a Hemocultura previa ao Antibiótico, a coleta de creatinina e plaquetas e a realização de pressão venosa central no paciente sem ventilação mecânica ( $p>0,05)$. A relação dos possíveis desfechos em até 14 dias (óbito, internação e alta) e entre 15 a 30 dias e suas associações com a adesão aos manejos preconizados pelo pacote de atendimento para sepse e choque séptico estão descritos na Tabela 4. 
11 | Duarte RT, Oliveira APA, Moretti MMS, Urbanetto JS

Tabela 4: Associação da adesão às ações relacionadas ao pacote de atendimento para sepse e choque séptico com os desfechos apresentados por pacientes em uma unidade de emergência no ano de 2014 ( $\mathrm{n}=312)$. Porto Alegre, RS, Brasil, 2015.

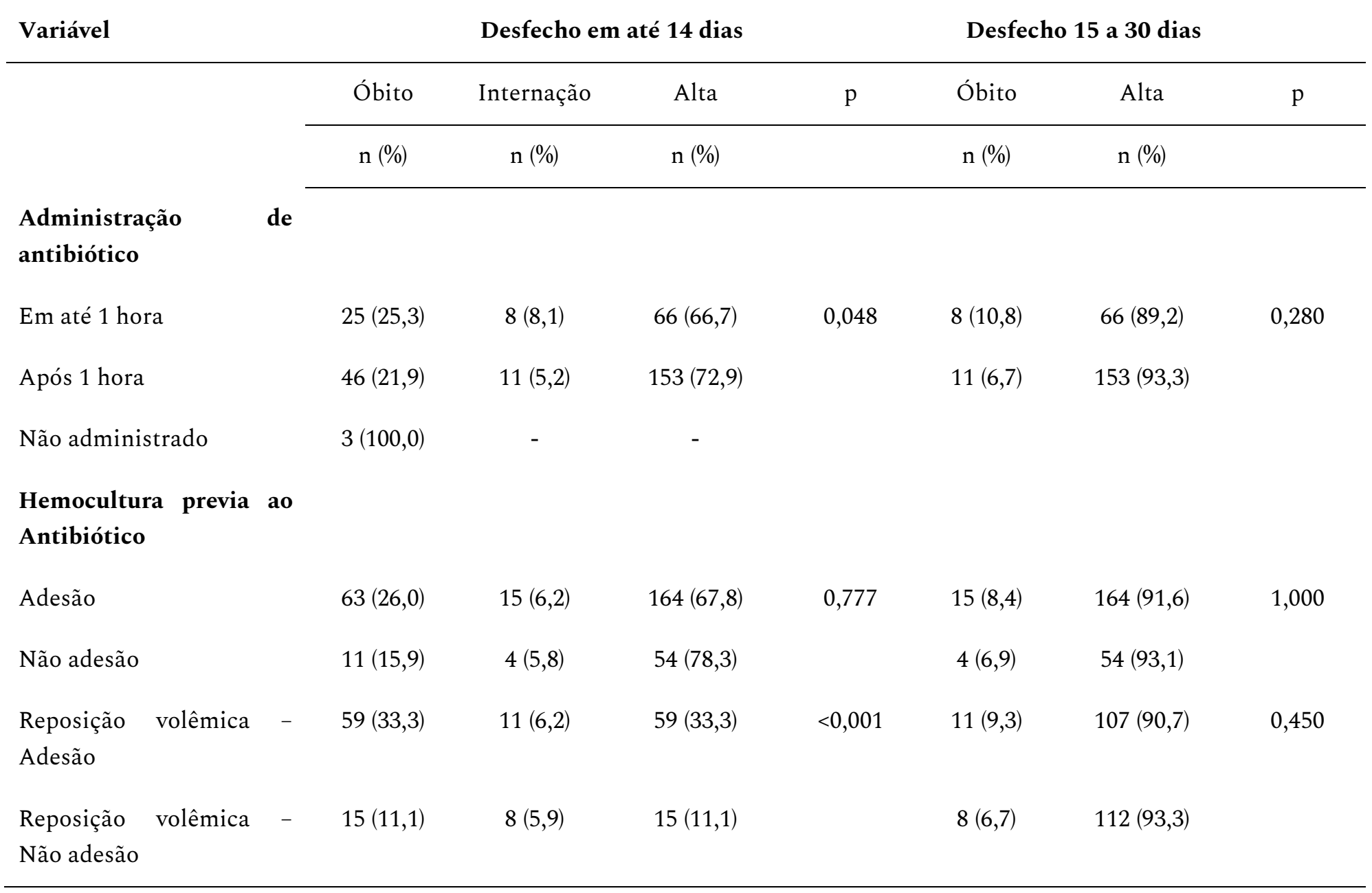

\section{Discussão}

Os dados relacionados às questões de faixa etária, sexo fatores clínicos e a gravidade da sepse apresentados pelos pacientes deste estudo assemelham-se aos dados expostos por demais autores, em que a média de idade foi de 61,1 anos $( \pm 21,6)$ e dentre as comorbidades apresentadas, a hipertensão foi a patologia mais prevalente, seguida por diabetes e doenças cardiovasculares, divergindo apenas no que se refere a prevalência do sexo, pois em um dos estudos a prevalência foi discretamente masculina. ${ }^{6-8}$ 
Associação dos fatores demográficos, clínicos e do manejo terapêutico ao desfecho de pacie... $\mid 12$

Dados encontrados em relação à classificação por gravidade estão em consonância com os dados encontrados em alguns estudos, pois $66,6 \%$ dos pacientes assistidos em emergências apresentavam Sepse e 33,4\%, Choque séptico. ${ }^{6-8} \mathrm{O}$ hospital em que esta pesquisa foi realizada tem um perfil de atendimento clínico/cirúrgico e isso pode refletir em variações pequenas no índice de gravidade em relação ao restante do país.

Acerca das associações relacionadas à faixa etária, recentemente nos Estados Unidos, identificou que pacientes idosos ( $\geq 65 \mathrm{anos}$ ) foram responsáveis por $64 \%$ dos casos de sepse, apresentando maior associação com foco pulmonar e taxa de letalidade maior. A idade aumentada estava associada ao período de hospitalização maior e posterior óbito, ${ }^{9}$ assim como os dados obtidos nesse estudo.

Em relação ao sexo em associação com a mortalidade, identificou associação limítrofe com o D14 ( $\mathrm{p}=0,059)$ e associação com o D15 a 30. Foi identificada maior associação entre sexo feminino e alta em D14 e D15 a 30 e, no sexo masculino, maior associação a óbito. Em estudo publicado em 2010, encontraram dados diferentes, no qual mulheres apresentaram maior probabilidade de mortalidade hospitalar do que homens. ${ }^{10}$

Pacientes na faixa etária de 18 a 56 e 72 a 95 anos apresentaram mais casos de sepse, enquanto pacientes na faixa etária de 57 e 71 anos incidência maior de casos de choque séptico. O sexo também foi relevante, homens com mais diagnóstico de choque séptico em comparação a mulheres. ${ }^{9-11}$ Não existe descrição específica desses dados, porém pode-se inferir que esta associação é coerente tendo em vista que a maioria dos pacientes que evoluíram ao óbito também eram idosos, homens e foram diagnosticados com choque séptico.

Sobre as comorbidades que associaram-se ao desfecho, observou-se que pacientes com histórico de AVE, cirrose e cardiopatias tiveram associação com óbito no período de até D14, diferentemente dos dados encontrados na literatura que sugerem comorbidades como diabéticos, hipertensos, imunossupressos e câncer como as principais patologias relacionadas a 
13 | Duarte RT, Oliveira APA, Moretti MMS, Urbanetto JS

gravidade da sepse e ao desfecho. ${ }^{12-13}$ Este achado indica que outras comorbidades também parecem ter um papel importante no desfecho dos pacientes com sepse ou choque séptico, e merecem aprofundamento em estudos relacionados a esta temática.

Em relação aos pacientes com cirrose hepática, evidenciou-se associação com o desfecho óbito e isso é descrito na literatura associado ao fato de que esse tipo de paciente é mais suscetível a infecções devido a uma maior translocação bacteriana relacionada a disfunção hepática. ${ }^{14}$ Pacientes cirróticos possuem duas vezes mais chances de evoluírem para óbito por sepse do que pacientes não cirróticos, apresentando taxa de mortalidade de $70 \%$ relacionada a choque séptico e $40 \%$ a sepse. ${ }^{15-16}$

Ainda analisando a associação das variáveis com os desfechos, pode-se inferir que o foco pulmonar, mesmo sendo o mais prevalente, não apresentou associação com os desfechos, assim como os demais focos infecciosos analisados neste estudo. ${ }^{17}$ Em contrapartida, a gravidade da sepse apresentou associação entre os casos de choque séptico e óbito no D14 e D15 a 30, enquanto os pacientes com diagnóstico de sepse evoluíram para alta hospitalar em ambos os períodos. $^{7}$

Os sinais de alerta referentes à alteração da temperatura (hipotermia), taquipneia e hipotensão apresentaram maior associação com desfechos relacionados ao óbito nos períodos de até D14 dias e de D15 a 30. Na literatura consultada não há descrição de associação direta desses dados com o desfecho e sim com o conjunto de sinais e disfunções utilizados para identificação precoce da sepse reduzindo em $16 \%$ as chances de óbito. ${ }^{17}$

Todas as disfunções orgânicas avaliadas manifestaram associação com os desfechos, sendo que a alteração dos parâmetros avaliados esta relacionada com o óbito dos pacientes. De acordo com uma pesquisa realizada em Pernambuco, as principais disfunções orgânicas associadas ao óbito foram plaquetopenia, RNI $>1,5$ e lactato $>4 \mathrm{mmol} / \mathrm{L} .{ }^{18}$ 
Associação dos fatores demográficos, clínicos e do manejo terapêutico ao desfecho de pacie... $\mid 14$

Outro aspecto relevante encontrado foi a associação das ações relacionadas ao pacote de atendimento ao paciente séptico com os desfechos em ambos os períodos de tempo. Destaca-se a tendência de associação ao desfecho óbito nos pacientes que receberam manejo indicado pelo pacote de sepse. Entretanto, essa incongruência se explica pelo fato de que os pacientes que receberam as medidas do pacote de atendimento serem os mais graves, com diagnóstico de choque séptico. Explorando um pouco mais alguns itens do pacote de medidas para o manejo, como a existência de associação entre eles e os desfechos propostos.

Houve associação entre administração do antibiótico em até uma hora após o diagnóstico com a alta do paciente e a não administração de antibiótico esteve associada com o óbito (em D14). Estas variáveis não se associam com o desfecho no período de D15 a 30. Nesse estudo não foi avaliado o tempo médio de administração do antibiótico. Uma pesquisa recente demonstrou que as recomendações realizadas pela Surving Sepsis Campaing em relação à administração de antibiótico até uma hora após diagnóstico não são suportadas pelas evidências clínicas disponíveis atualmente em relação à mortalidade. ${ }^{19}$

A reposição volêmica foi realizada em $41,3 \%$ dos pacientes e a coleta de lactato sérico em 71,4\%. Esse dado demonstra déficit na assistência realizada e desacordo com as recomendações com grau de evidência $\mathrm{A}$, já que $44 \%$ dos pacientes que chegam à emergência e são incluídos no protocolo de sepse apresentam hipotensão, alteração de temperatura, taquipnéia e taquicardia, necessitando dessas ações para o manejo adequado. ${ }^{20}$

Os dados coletados nesse estudo demonstram que pacientes com diagnóstico de sepse e o desfecho internação em até D14, bem como o desfecho alta entre D15 a 30 estão associados. Em contrapartida, pacientes com diagnóstico de choque séptico estão associados a desfecho óbito tanto em D14 como no período de D15 a 30. Os pacientes em choque séptico tendem a ser manejados de maneira diferenciada na emergência, sendo realizada a maioria dos exames laboratoriais possíveis e instalação de dispositivos invasivos como SVD, acesso venoso central e 
15 | Duarte RT, Oliveira APA, Moretti MMS, Urbanetto JS

PVC. Não é possível inferir, com certeza, se o manejo recebido pelos pacientes do estudo foi incorreto ou não, pois se sabe que na prática clínica nem todo paciente em sepse necessita receber determinados dispositivos avaliados nas condutas referentes ao manejo, como acesso venoso central, ventilação mecânica invasiva, entre outros.

Estudos recentes vêm demonstrando que o tratamento realizado através do Earl goaldirected therapy (EGDT), no Brasil ${ }^{21}$ conduzido como estabilização hemodinâmica precoce, apresenta associação a redução de mortalidade em pacientes sépticos. Diferentemente do que é encontrado na literatura de países de primeiro mundo, onde essa metodologia não implica na redução da mortalidade. No entanto, influenciam diretamente na redução de custos hospitalares. ${ }^{21-22}$ Outros estudos relacionaram o impacto da adesão do bundle da Surving Sepsis Campaign, incluindo pacotes de 3 e 6 horas, encontraram associação entre o manejo do paciente em sepse e choque séptico por meio de bundles a $25 \%$ de risco relativo de redução da mortalidade. ${ }^{23} \mathrm{O}$ manejo precoce do paciente séptico, além de impactar na mortalidade, influencia diretamente na redução do tempo de internação desses pacientes, bem como na redução de custos relacionados à assistência. ${ }^{23}$

\section{Conclusão}

Foi possível, inferir que há associação entre o perfil demográfico do paciente, suas comorbidades, as disfunções orgânicas apresentadas pelo mesmo e o manejo terapêutico realizado com a gravidade da sepse e com o desfecho clínico. Apenas os dados referentes ao foco infeccioso não apresentaram associação com o desfecho.

Além disso, identificar que mesmo com a campanha da Surving Sepsis Campaing em vigor, ainda existem profissionais que não aderem ao pacote de medidas preconizadas. Muitas vezes isso acontece pelos fatores que atrapalham a clínica da sepse e pela descrença no diagnóstico, que faz com que o pacote de exames para identificação de várias disfunções 
Associação dos fatores demográficos, clínicos e do manejo terapêutico ao desfecho de pacie... I 16

orgânicas não seja realizado, impactando na evolução da gravidade de sepse, no desfecho e, por consequência, no tempo de internação e nos custos relacionados à assistência desses pacientes.

O fato de haver associação com óbito e algumas ações do manejo como: coleta de todos os exames laboratoriais do pacote, cateter venoso central e PVC, não permite concluir que o manejo recebido por esses pacientes tenha sido deficitário, ou que estas ações estão associadas a um pior prognóstico, mas sim, que provavelmente estes pacientes estivessem apresentando um quadro de maior gravidade e comprometimento orgânico. Pela grande heterogeneidade apresentada pelos pacientes sépticos, conclui-se que ainda são necessários estudos para reconhecimento do perfil dos pacientes com sepse nas emergências brasileiras e os fatores que contribuem ou não para a diminuição da morbimortalidade resultante deste agravo. Além disso, mais estudos proporcionariam a identificação de evidências clínicas para o manejo preconizado e talvez assim, maior adesão ao pacote de medidas.

Atualmente o ILAS está desenvolvendo um estudo sobre pacientes sépticos atendidos nas emergências brasileiras denominado Sepsis Prevalence Assessment Database (SPREAD PS), entretanto os resultados ainda não foram divulgados. Espera-se com essa pesquisa sanar algumas deficiências desse estudo como a escassez de publicações brasileiras referentes ao perfil do paciente séptico, como também a falta de registros de atendimentos no prontuário dos pacientes.

\section{Referências}

1. Rhodes A, Evans LE, Alhazzani W, Levy M, Antonelli M, Ferrer R, et al. Surviving Sepsis Campaign: international guidelines for management of sepsis and septic shock. Crit Care Med [Internet]. 2017 $\begin{array}{llll}\text { [acesso em } & 2017 & \text { maio } & \text { 43(3):304-77. }\end{array}$ Disponível em: https://journals.lww.com/ccmjournal/Fulltext/2017/03000/Surviving_Sepsis_Campaign___I nternational.15.aspx

2. Instituto Latino Americano de Sepse (ILAS). Implementação de protocolo gerenciado de sepse: protocolo clínico [Internet]. São Paulo; 2016; [acesso em 2017jun 28]. Disponível em: http://www.ilas.org.br/assets/arquivos/ferramentas/protocolo-de-tratamento.pdf 
3. Singer M, Deutschman CS, Seymour CW, Shankar-Hari M, Annane D, Bauer M, et al. The third international consensus definitions for sepsis and septic shock (Sepsis-3). JAMA [Internet]; 2016 [acesso em 2017 jun 26];315(8):801-10. Disponível em: https://jamanetwork.com/journals/jama/fullarticle/2492881

4. Machado FR, Assunção MSC, Cavalcanti AB, Japiassú AM, LCP Azevedo, Oliveira MC. Chegando a um consenso: vantagens e desvantagens do Sepsis 3 considerando países de recursos limitados. Rev Bras Ter Intensiva [Internet]. 2016 [acesso em 2017 jun 26];28(4):361-5. Disponível em: http://www.scielo.br/pdf/rbti/v28n4/0103-507X-rbti-28-04-0361.pdf

5. Barros LLS ,Maia CSFM, MC Monteiro. Fatores de risco associados ao agravamento de sepse em pacientes em Unidade de Terapia Intensiva. Cad Saúde Colet [Internet]. 2016 [acesso em $2018 \mathrm{dez}$ 04];24(4):388-96. Disponível em: http://www.scielo.br/scielo.php?pid=S1414462X2016000400388\&script=sci_abstract\&tlng=pt

6. Instituto Latino Americano de Sepse (ILAS). Campanha "sobrevivendo à Sepse" - relatório trimestral [Internet]. São Paulo [acesso em 2005 nov 14]. Disponível em: http://www.sepsisnet.org

7. Farias LL, Pinheiro Júnior FML, Braide ASG, Macieira CL, Araújo MVUM, Viana MCC, et al. Perfil clínico e laboratorial de pacientes com sepse, sepse grave e choque séptico admitidos em uma unidade de terapia intensiva. Rev Saúde Públ Santa Cat [Internet]. 2013 jul-set [acesso em 2015 nov 05];6(3):50-60. Disponível em: http://repositorio.ufc.br/ri/bitstream/riufc/8345/1/2013_art_fmlpinheirojunior.pdf

8. Ranzini OT, Monteiro MB, Ferreira EM, Santos SR, Machado FR, Noritomi DT. Reclassificando o espectro de pacientes sépticos com o uso do lactato: sepse grave, choque críptico, choque vasoplégico e choque disóxico. Rev Bras Ter Intensiva [Internet]. 2013 [acesso em 2015 nov 10];25(4):270-8. Disponível em: http://www.scielo.br/pdf/rbti/v25n4/0103-507x-rbti-25-04-0270.pdf

9. Palomba H, Corrêa TD, Silva E, Pardini A, Assuncao MSC. Comparative analysis of survival between elderly and non-elderly severe sepsis and septic shock resuscitated patients. Einstein [Internet]. 2015 acesso em 2015 set 05];13(3):357-63. Disponível em: https://www.ncbi.nlm.nih.gov/pmc/articles/PMC4943779/pdf/1679-4508-eins-13-3-

0357.pdf doi:10.1590/S1679-45082015AO3313

10. Moura JM, Bertolli ES, Pereira RM, Frutuoso IS, Werneck AL, Contrin LM. Diagnóstico de sepse em pacientes após internação em unidade de terapia intensiva. Arq Ciênc Saúde [Internet]. 2017 [acesso em 2018 set 05];24(3):55-60. Disponível em: http://www.cienciasdasaude.famerp.br/index.php/racs/article/view/675/711

11. Baracho NCV, Lopes GF, Araujo TD, Buissa T, Yano WK. Fatores de risco associados à mortalidade em pacientes com sepse grave e choque séptico na unidade de terapia intensiva de um hospital escola do sul de Minas Gerais. Rev Ciênc Saúde. 2011;1(1):1-6. 
Associação dos fatores demográficos, clínicos e do manejo terapêutico ao desfecho de pacie... I 18

12. Martin G. Sepsis, severe sepsis and septic shock: changes in incidence, pathogens and outcomes. Expert Rev Anti Infect Ther [Internet] 2012 [acesso em 2015 dez 08];10(6):701-6. Disponível em: http://www.ncbi.nlm.nih.gov/pmc/articles/PMC3488423/pdf/nihms-413357.pdf

13. Branco J. Sepsis no doente com cirrose hepática [monografia]. Sintra (Portugal): Hospital Professor Doutor Fernando da Fonseca; 2013. 37p.

14. Carrola P, Militão I, Presa J. Infecções bacterianas no doente com cirrose hepática. GE J Port Gastrenterol [Internet]. 2013 [acesso em 2018 set 05];20(2):58-65. Disponível em: http://www.scielo.mec.pt/pdf/ge/v20n2/v20n2a03.pdf

15. Juncal VR, Britto Neto LA, Camelier AA, Messender OHC, Farias AMC. Impacto clínico do diagnóstico de sepse à admissão em UTI de um hospital privado em Salvador, Bahia. J Bras Pneumol [Internet]. 2011 [acesso em 2015 dez 08];37(1):85-92. Disponível em: http://www.scielo.br/pdf/ibpneu/v37n1/v37n1a13.pdf

16. Barros LLS ,Maia CSF, Monteiro MC. Fatores de risco associados ao agravamento de sepse em pacientes em Unidade de Terapia Intensiva. Cad Saúde Colet [Internet]. 2016 [acesso em 2018 set 05];(24)4:388-96. Disponível em: http://www.scielo.br/pdf/cadsc/v24n4/1414-462X-cadsc-24-4-388.pdf

17. Koury JCA, Lacerda HR, Barros Neto AJ. Fatores de risco associados à mortalidade em pacientes com sepse em unidade de terapia intensiva de hospital privado de Pernambuco. Rev Bras Ter Intensiva [Internet]. 2007 [acesso em $2015 \quad \mathrm{dez}$ 08];19(1):23-30. Disponível em: http://www.scielo.br/scielo.php?script=sci_arttext\&pid=S0103-507X2007000100003

18. Sterling SA, Miller WR, Pryor J, Puskarich MA, Jones AE. The impact of timing of antibiotics on outcomes in severe sepsis and septic shock: a systematic reviewand meta-analysis. Crit Care Med [Internet]. 2015 [acesso em 2015 dez 08];43(9):1907-15. Disponível em: http://www.ncbi.nlm.nih.gov/pubmed/26121073

19. ARISE Investigators, ANZICS Clinical Trials Group; Peake SL, Delaney A, Bailey M, Bellomo R, et al. Goal-directed resuscitation for patients with early septic shock. N Engl J Med [Internet]. 2014 [acesso em 2015 dez 08];371(16):1496-506. Disponível em: http://www.ncbi.nlm.nih.gov/pubmed/25272316

20. Wawrzeniak IC, Loss SH, Moraes MCM, De La Vega FL, Victorino JA. Could a protocol based on early goal-directed therapy improve outcomes in patients with severe sepsis and septic shock in the Intensive Care Unit setting? Indian J Crit Care Med [Internet]. 2015 [acesso em 2017 jun 28];19(3):159-65. Disponível em: https://www.ncbi.nlm.nih.gov/pmc/articles/PMC4366915/

21. Mouncey PR, Osborn TM, Power GS, Harrison DA, Sadique MZ, Grieve RD, et al. Trial of early, goaldirected resuscitation for septic shock. N Engl J Med [Internet]. 2015 [acesso em 2015 dez 08];372(14):1301-11. Disponível em: https://www.ncbi.nlm.nih.gov/pubmed/25776532 
19 | Duarte RT, Oliveira APA, Moretti MMS, Urbanetto JS

22. Levy MM, Rhodes A, Phillips GS, Townsend SR, Schorr CA, Beale R, et al. Surviving sepsis campaign: association between performance metrics and outcomes in a 7.5-year study. Crit Care Med [Internet]. 2015 [acesso em 2015 nov 08];43(1):3-12. Disponível em: http://www.ncbi.nlm.nih.gov/pubmed/25275252

23. Noritomi DT, Ranzani OT, Monteiro MB, Ferreira EM, Santos SR, Leibel F, et al. Implementation of a multifaceted sepsis education program in an emerging country setting: clinical outcomes and costeffectiveness in a long-term follow-up study. Intensive Care Med [Internet]. 2014 [acesso 2015 dez 08];40(2):182-91. Disponível em: http://www.ncbi.nlm.nih.gov/pubmed/24146003

\section{Autor correspondente}

Rayssa Thompson Duarte

E-mail: rayssatd@hotmail.com

Endereço: Rua Sarmento Leite, 245, Centro, Porto Alegre, RS, Brasil.

CEP: $90050-170$

\section{Contribuições de Autoria}

1 - Rayssa Thompson Duarte

Contribuições: - Concepção e planejamento do projeto de pesquisa; obtenção, análise e interpretação dos dados; redação e revisão crítica.

2 - Ana Paula Amestoy de Oliveira

Contribuições: - Concepção e planejamento do projeto de pesquisa; obtenção, análise e interpretação dos dados; redação e revisão crítica.

3 - Miriane Melo Silveira Moretti

Contribuições: - obtenção, análise e interpretação dos dados; e revisão crítica.

3 - Janete de Souza Urbanetto

Contribuições: - Concepção e planejamento do projeto de pesquisa; análise e interpretação dos dados; e revisão crítica.

\section{Como citar este artigo}

Duarte RT, Oliveira APA, Moretti MMS, Urbanetto JS . Associação dos fatores demográficos, clínicos e do manejo terapêutico ao desfecho de pacientes sépticos atendidos em uma emergência hospitalar. 2019 [Acesso em: Anos Mês Dia];vol e42: Px-P19. DOI:https://doi.org/10.5902/2179769234413 